DOI: $10.12731 / 2306-1561-2013-4-37$

\title{
THE SITUATIONAL APPROACH TO MANAGEMENT: ORIGINS AND PROSPECTS
}

\section{Mironov V.V., Konev K.A., Shakirova G.R.}

\section{Abstract}

The article is devoted to a brief review of well-known methodologies situational management. Discusses the various terminological aspects of the situational approach in relation to the different branches of science and production. The article considers the prospects of situational management in the context of the development of modern information technologies.

Keywords: situational approach; situational management; situation; modeling; management.

\section{УДК 004.9}

\section{СИТУАЦИОННЫЙ ПОДХОД К УПРАВЛЕНИЮ: ИСТОКИ И ПЕРСПЕКТИВЫ}

\section{Миронов В.В., Конев К.А., Шакирова Г.Р.}

\section{Аннотация}

Статья посвящена краткому обзорному анализу известных методологий ситуационного управления. Обсуждаются различные терминологические аспекты ситуационного подхода применительно к различным отраслям науки и производства. Рассматриваются перспективы ситуационного управления в контексте развития современных информационных технологий.

Ключевые слова: ситуачионный подход; ситуационное управление; ситуация; моделирование; управление.

В настоящее время сложно переоценить значение управления в различных областях деятельности. Характерной особенностью управления любыми объектами является необходимость принятия решений, направленных на достижение определенных целей управления.

Сложность объектов управления усиливается невозможностью их строгого описания, исключая применение известных аналитических, логико-эвристических и интеллектуальных подходов к управлению, ориентированных на строго формализуемые системы. На первый план выходят методы ситуационного управления, 
позволяющие принять решение на основе сопоставления текущей ситуации заданному набору возможных решений.

При таком подходе понятие ситуации является исходным базовым понятием, построение множества ситуаций, возможных в системе управления, рассматривается как начальный конструктивный шаг разработки системы, который должны выполнять разработчики на основе своих знаний и опыта, исходя из своего понимания целей и условий функционирования системы [1].

В настоящее время ситуационный подход активно развивается в самых разных научных областях. Каждое из таких направлений имеет свой подход и методологическую базу. Обсудим наиболее известные научные подходы к пониманию ситуаций.

Своя трактовка термина «ситуация» сформулирована в рамках приложения ситуационного подхода к управлению сложными системами [2]. Ситуационное управление сложными системами, с точки зрения данного подхода, предполагает последовательное преобразование ситуации в результате принятия некоторого решения. Если для текущей ситуации $s(t)$ принимается соответствующее решение, то происходит переход к ситуации $s(t+1)$.

Число ситуаций, которые возможны при управлении сложными системами, может быть очень большим, поэтому важно обеспечить адекватное разбиение ситуаций на группы. Теория ситуационного управления предлагает два варианта такого анализа.

Первый способ определяется тем, что между числом допустимых решений $\mathrm{R}$ и числом состояний уровня $\mathrm{S}$ имеется соотношение $|S|>>R \mid$. Для сходных ситуаций могут быть применены одинаковые решения. Достаточно разбить множество ситуаций на группы в соответствии с применимыми к ним решениями. В известных работах (например, [2]) такое разбиение носит название обобщения или классификации. Базовым классификационным признаком является применимость к ситуации конкретного решения, а задача принятия решений сводится к обобщению (структурированию) ситуаций. Определяется класс, к которому принадлежит текущая ситуация ситуации $s(t)$. Затем выбирается команда управления, соответствующая этому классу, по которой ситуация $s(t)$ преобразуется в ситуацию $s(t+1)$ и т. д. (рисунок 1$)$.

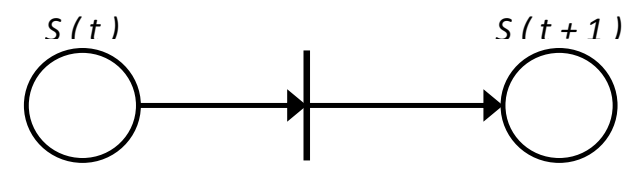

Анализ применимости,

выбор класса, выбор

решения

\section{Рисунок 1 - Механизм смены состояний в ситуационном управлении}

Функционирование сложной системы расценивается как процесс изменения пространственно-временных отношений между ее элементами. Такой процесс 
целесообразно представлять в форме дискретных ситуационных сетей (ДСС), т.е. графов, вершинам которых соответствуют элементы системы, а ребрам - связи между ними. При этом ситуацией в момент времени $t$ является подграф ДСС, вычленяемый в момент времени $t$.

Еще один способ обобщения ситуаций в соответствии с множеством допустимых решений предполагает использование операций теоретико-множественного пересечения. В его основе лежит множество правил экстраполяции ситуаций. Применение теоретико-множественного пересечения к заданному множеству ситуаций дает множество обобщенных ситуаций, которое, в свою очередь, сопоставляется с множеством решений.

Один из базовых подходов к рассмотрению понятия «ситуации» приведен в трудах по ситуационному управлению Д.А. Поспелова [3]. В его работах рассматриваются основные принципы ситуационного подхода при организации управления объектами различной природы.

Ситуационное управление целесообразно только применительно к объектам с неопределенной или недостаточно изученной структурой и набором свойств, таким объектам и процессам, для которых можно единожды создать и в дальнейшем наращивать некоторую базу знаний - решений, принимаемых в той или иной конкретной ситуации.

В основе ситуационного управления лежат так называемые семиотические модели, которые можно рассматривать как «слабоформальные» модели, задаваемые не множеством математических формул и соотношений, а некоторым набором утверждений - аксиом. Такие знаковые системы не обладают жестким синтаксисом и семантикой, отражающей изменчивость рассмотрения одной и той же ситуации с разных точек зрения, в разном контексте. Иными словами, с помощью знаковых (семиотических) моделей одна и та же ситуация может быть представлена по-разному. К знаковым относятся как вербальные (набор предложений-утверждений), так и графические утверждения (различные графические модели).

В этом контексте понятие ситуации определено на двух уровнях - текущей и полной ситуаций. Текущая ситуация объекта управления представляет собой совокупность данных о структуре и функционировании объекта управления в конкретный момент времени. Полная ситуация - это объединение текущей ситуации, знаний о состоянии системы управления в конкретный момент времени и знаний о методологии управления.

Полная ситуация $S$ дает представление о том, как из текущей ситуации $S_{l}$ с помощью доступных механизмов (способов воздействия на объект управления) $U$ перейти к ситуации $S_{2}$. Таким образом, полную ситуацию можно представить элементарным соотношением:

$$
S: S_{1} \longrightarrow S_{2}
$$


Такое правило преобразования носит название логико-трансформационного или корреляционного правила.

В данном контексте интерес представляют различные способы описания ситуаций. В работах Поспелова в качестве языка ситуационного управления предлагаются дискретные ситуационные сети (ДСС). Фактически в таких сетях имеет место экстраполяция развития ситуаций и оценка влияния тех или иных решений по управлению. Развитие ситуации представляется в виде ориентированного графа, вершинами которого являются различные ситуации, а дугами - переходы между ситуациями. ДСС предусматривает множественные переходы между ситуациями выбор единственного решения при этом определяется специально введенными правилами (к примеру, расстановкой приоритетов переходов). ДСС функционирует в дискретные такты - в каждый момент времени порождается новая полная ситуация: происходит смена текущей ситуации объекта управления, для которой определены собственные механизмы обработки.

В действительности ДСС рассматривается как определённый сценарий: вершинам соответствуют факты, а дугам - связи. Связи могут служить отношением типа: причина - следствие, часть - подчасть, цель - подцель и т. п. В таком контексте ДСС больше относится к классу экспертных систем, чем способу представления развития ситуации это скорее набор правил, база знаний, чем описания типа «ситуация - переход ситуация». К однотипным ситуациям могут быть применены несколько альтернативных решений. Иными словами, текущая ситуация порождает несколько полных ситуаций, каждый экземпляр которой определяет одно одношаговое решение.

Важной особенностью такого класса систем является то, что число различных полных ситуаций обычно весьма велико, а число решений конечно и невелико. Например, находясь за рулем автомобиля, водитель воспринимает множество полных ситуаций, определяемых внешней обстановкой на дороге и состоянием автомобиля. А число принимаемых им одношаговых решений невелико.

Таким образом, возникает проблема выбора единственного решения из множества возможных в данной текущей ситуации. Поспелов в своей книге [3] предлагает дополнение системы управления специальным элементом, классификатором, который по выбранным признакам относит ситуацию к определенному классу и экстраполирует множество доступных при этом решений. В этом плане множество полных ситуаций представляется в виде конечного числа классов.

Тогда задача классификации формулируется следующим образом. На множестве определённых ситуаций $\left\{Q_{i}\right\}$ следует отыскать такой способ разбиения их на классы, при котором каждый класс Qi имел бы в рамках рассматриваемой модели управления адекватную интерпретацию процедуры поиска решений по управлению объектом. Иными словами, имеет место двухуровневая классификация:

- классификация текущих ситуаций;

- классификация полных ситуаций (возможных решений в контексте текущей ситуации). 
Классификация ситуаций носит иерархический характер - в процессе обобщения ситуаций и появления классов ситуаций возникает естественная иерархия, определяемая вхождением одних классов в другие. Выделяются два базовых классификационных признака - по признакам и по структурам.

Обобщение может происходить на разных этапах управления, поэтому исходные и обобщенные описания ситуаций образуют иерархическую структуру, в каждом слое которой находятся описания, полученные из исходных с помощью процедур обобщения.

Таким образом, начальный уровень образуют исходные данные, полученные о ситуациях. Следующие уровни формируются с помощью применения процедур обобщения к описаниям, полученным на предшествующих уровнях. Так возникает так называемый «слоеный пирог» описания ситуаций.

Несколько иной подход к определению понятия «ситуация» дается в контексте управления техническими системами [4]. Такое решение носит в большей степени физический характер и рассматривается применительно к явлению приспособляемости движения, т. е. возможности достижения цели физическим телом при воздействии на него окружающей среды.

При таком подходе принципиальное значение имеют следующие понятия (рисунок 2):

- обстановка - это все внутренние и внешние явления, которые нельзя отнести к управлению;

- событие - любое изменение обстановки. Изменение обстановки предполагает переход от одного ее класса к другому. Определяющим фактором такого изменения является необходимость принятия решения. Так, если при переходе обстановки от А к В или обратно требуется принимать решение о дальнейшем управлении, то А и В относятся к различным классам обстановок. Событие можно позиционировать как процесс принятия решения;

- решение - это, с теоретической точки зрения, переключение условных задач, целей, программ, законов управления и т. п. Систему управления можно представить состоящей из элементарных блоков, которые в зависимости от обстановки переключаются в различных комбинациях. Такие сочетание блоков - элементарные системы - и являются решениями.

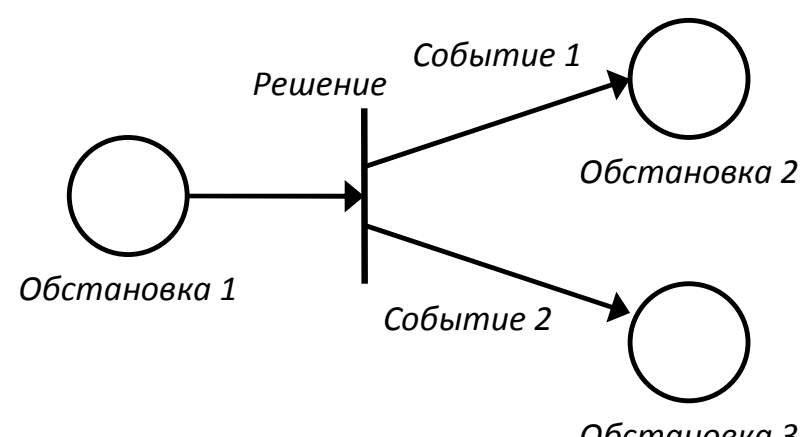

Рисунок 2 - Ситуационное управление техническими системами 
Происходящее на дороге - это обстановка для управления автомобилем. В соответствии с этим обстановка делится на внешнюю, определяемую явлениями или событиями вне системы управления, и внутреннюю, задаваемую внутренними параметрами системы управления, но не относящимися к самому процессу управления.

Событием в том же примере может считаться изменение внутренней или внешней обстановки: появление пешехода, встречного автомобиля, поломка двигателя и пр. В соответствии с данным подходом ситуация задается совокупностью класса обстановки и принятого решения. Согласно такому представлению, классификация ситуации может рассматриваться как классификация ее составляющих.

События, относительно которых нам неизвестно, где или когда они могут произойти, относятся к классу непредсказуемых. События, относительно которых нам достоверно известно, когда и где они произойдут, относятся к классу предсказуемых. Предсказуемые события порождают предсказуемую обстановку. Если среди группы событий есть хотя бы одно непредсказуемое, то вместе они приводят к непредсказуемой обстановке. Кроме того, обстановка может быть ожидаемой и неожидаемой. Ожидаемые классы определяют ту обстановку, на которую рассчитывается система управления, неожидаемые - обстановку, на работу в которой система не рассчитывается.

В рамках уфимской школы ситуационного управления предложена несколько иная трактовка понятия ситуации [5 - 8]. Специфика данного подхода состоит в рассмотрении ситуации на двух уровнях:

- на макроуровне: данная конкретная ситуация рассматривается не обособленно, а в ряду всех возможных ситуаций;

- на микроуровне: ситуация рассматривается автономно, с точки зрения ее «инфраструктуры», внутренней сущности.

Пусть известна динамическая система, заданная в общепринятой форме «входсостояние-выход». В ней рассматривается совокупность текущих характеристик, представленная элементами: текущий момент времени; текущее состояние; входные воздействия (управляющие и возмущающие), заданные для текущего и будущего моментов времени, которая носит название микроситуации. При этом ситуация представляет собой набор микроситуаций. Фактически микроситуации можно рассматривать как определенные этапы, состояния ситуации, т.е. они задают эволюцию, развитие некоторой ситуации.

Тогда, с точки зрения теории систем [9], свойства ситуации определяются свойствами составляющих ее микроситуаций. Совокупность характеристик, составляющих микроситуацию, определяет выходные характеристики и состояния системы, как в текущий, так и в будущие моменты времени.

Временная составляющая микроситуаций в частности и ситуации вообще существенна для нестационарных систем, свойства которых меняются во времени. Иными словами, при наличии некоторого фиксированного момента времени рассмотрение ситуации возможно в двух аспектах - ситуация до наступления заданного момента времени и ситуация после него. 
Прогнозируемость ситуации выражается в том, что каждая микроситуация не просто описывает некоторое состояние, а рассматривает его в целом, вместе с временным параметром, возмущающими и управляющими воздействиями. Последние как раз и показывают, к чему может привести переход из данного состояния в результате принятия какого-то решения (управляющие воздействия) или воздействия внешней среды (возмущающие).

Ситуации должны быть сконструированы так, чтобы имелась возможность рассматривать их в задаче управления как единые объекты, не принимая во внимание их внутреннюю «микроситуационную» структуру. В этом плане к микроситуациям принимается требование гомогенности, они должны быть эквивалентны друг другу в смысле некоторого критерия.

Рассматриваемый ситуационный подход ставит своей целью исследование одной из групп ситуаций - критических ситуаций. С этой целью предложен оригинальный термин, отражающий суть этого явления. Критическая ситуация понимается как такое изменение характеристик системы в результате нарушений (отказов, внешних воздействий, ошибок управления), которое в случае непринятия своевременных и целенаправленных мер может привести к опасным последствиям. С учетом сказанного важно отметить, что качественная смена ситуации является результатом изменения микроситуации, переходом ее в новый класс.

Ситуационное управление предусматривает построение ситуационной модели процесса управления в виде совокупности управленческих ситуаций и их возможных переходов. Важен уровень рассмотрения ситуации - на макроуровне можно наблюдать только смену ситуаций в текущем процессе, на микроуровне - причину такого изменения или перехода.

Таким образом, рассмотренные подходы к трактовке понятия ситуации основываются на сходных интуитивных представлениях о ситуации, однако заметно различаются в конкретных определениях. По сути, каждое из них понимают ситуацию как совокупность некоторых внешних и внутренних условий, в которых функционирует объект управления.

Еще один существенный аспект - классификация ситуаций. Практически все рассмотренные подходы обуславливают необходимость классификации ситуации как ее разбиения на группы по множеству допустимых решений. В результате имеет место как сокращение числа возможных (и зачастую схожих) ситуаций, так и упрощение выбора решения по принадлежности ситуации некоторой группе. Каждый подход посвоему, в соответствии со своей спецификой видит такую классификацию, предоставляет свой набор базовых классификационных признаков.

\section{Список информационных источников}

[1] Ильясов Б. Г., Миронов В. В., Юсупова Н. И. Модели критических ситуаций при управлении техническими объектами: Препринт. Уфа: УНЦ РАН, 1996. 
[2] Клыков Ю. И. Ситуационное управление большими системами. М.: Энергия, 1974.

[3] Поспелов Д. А. Ситуационное управление. Теория и практика. М. Наука, 1986. $288 \mathrm{c.}$

[4] Коренев Г. В. Цель и приспособляемость движения. М.: Наука, 1974.

[5] Ильясов Б. Г., Миронов В. В., Юсупова Н. И. Модели предупреждения критических режимов управляемых объектов в условиях неопределенности: Препринт. Уфа: УНЦ РАН, 1994.

[6] Ильясов Б. Г., Миронов В. В., Юсупова Н. И. Модели критических ситуаций при управлении техническими объектами: Препринт. Уфа: УНЦ РАН, 1996.

[7] Миронов В.В., Юсупова Н.И., Шакирова Г.Р. Ситуационно-ориентированные базы данных: концепция, архитектура, xml-реализация // Вестник УГАТУ. Серия «Управление, вычислительная техника и информатика». 2010. Т. 14, № 2. С. 233244.

[8] Юсупова Н. И. Критические ситуации и принятие решений при управлении в условиях помех. Уфа: Гилем, 1997.

[9] Куликов Г.Г., Конев К.А., Суворова В.А. Теория систем и системный анализ: учеб. пособие. Уфа: УГАТУ, 2012. 159 с. 